\title{
Numerical and Experimental Investigation of Computed Tomography of Chemiluminescence for Hydrogen-Air Premixed Laminar Flames
}

\author{
Liang Lv, Jianguo Tan, and Yue Hu \\ Science and Technology on Scramjet Laboratory, College of Aerospace Science and Engineering, \\ National University of Defense Technology, Changsha, Hunan 410073, China \\ Correspondence should be addressed to Jianguo Tan; jianguotan@126.com
}

Received 20 May 2016; Revised 6 July 2016; Accepted 20 July 2016

Academic Editor: Xuesong Li

Copyright ( 2016 Liang Lv et al. This is an open access article distributed under the Creative Commons Attribution License, which permits unrestricted use, distribution, and reproduction in any medium, provided the original work is properly cited.

\begin{abstract}
Computed tomography of chemiluminescence (CTC) is a promising technique for combustion diagnostics, providing instantaneous 3D information of flame structures, especially in harsh circumstance. This work focuses on assessing the feasibility of CTC and investigating structures of hydrogen-air premixed laminar flames using CTC. A numerical phantom study was performed to assess the accuracy of the reconstruction algorithm. A well-designed burner was used to generate stable hydrogen-air premixed laminar flames. The $\mathrm{OH}^{*}$ chemiluminescence intensity field reconstructed from 37 views using $\mathrm{CTC}$ was compared to the $\mathrm{OH}^{*}$ chemiluminescence distributions recorded directly by a single ICCD camera from the side view. The flame structures in different flow velocities and equivalence ratios were analyzed using the reconstructions. The results show that the CTC technique can effectively indicate real distributions of the flame chemiluminescence. The height of the flame becomes larger with increasing flow velocities, whereas it decreases with increasing equivalence ratios (no larger than 1). The increasing flow velocities gradually lift the flame reaction zones. A critical cone angle of 4.76 degrees is obtained to avoid blow-off. These results set up a foundation for next studies and the methods can be further developed to reconstruct 3D structures of flames.
\end{abstract}

\section{Introduction}

The development of combustion theory and the improvement of advanced combustion equipment need detailed studies of flame structures. The application of laser-based diagnostic techniques, such as particle image velocimetry (PIV), planar laser induced fluorescence (PLIF), and planar laser induced Rayleigh scattering, can perform in situ, nonintrusive diagnostics of flame characteristics. These methods have advantages of obtaining planar information with high spatial and temporal resolution but at the cost of expensive and complex experimental devices, such as powerful lasers, ICCD cameras, and various optical components.

Compared to these laser-based diagnostic techniques, the chemiluminescence technique is relatively simple and cheap since there is no laser equipment. Chemiluminescence is a natural indicator of flame structures and combustion characteristics, because it intrinsically represents electronically excited radicals formed by exothermic reactions within reaction zones. The most common chemiluminescence is from $\mathrm{OH}(\mathrm{A}-\mathrm{X})$ with wavelength of $310 \mathrm{~nm}, \mathrm{CH}(\mathrm{A}-\mathrm{X})$ of $430 \mathrm{~nm}$, and Swan bands of $\mathrm{C}_{2}(\mathrm{~d}-\mathrm{a})$ between $470 \mathrm{~nm}$ and $550 \mathrm{~nm}$ [1]. These three types of chemiluminescence can indicate flame structures $[2,3]$, combustion characteristics of heat release rate [4], local equivalence ratio [5], and stability [6]. Although the chemiluminescence measurement is an effective method to study flame structures and combustion characteristics, it only captures integral results in light paths. This shortcoming that is unable to resolve spatial distributions greatly limits the application of this diagnostic technique. An effective way to solve the problem and obtain 3D flame structures is the combination of the chemiluminescence measurement with the computed tomography (CT) technique, which leads to a prospective tool for flame studies named as computed tomography of chemiluminescence (CTC). In 1988, Hertz and Faris [7] first used CTC to conduct a simple 2D reconstruction. 
Since then, studies on the CTC technique have been widely carried out.

The reconstruction algorithm and the experimental system are the foundations of the CTC technique. The optimization of them attracts worldwide interests. Denisova et al. [8] developed an algorithm based on local regularization and maximum entropy to meet the characteristics of the narrow emitting region and the great intensity gradient, which results in a better reconstruction even with few cameras and severe noises. Goyal et al. [9] further optimized the algorithm and obtained a new one using the maximization of entropy methodology, which significantly reduced errors as compared to $2 \mathrm{D}$ slice-by-slice reconstruction algorithms. Cai et al. [10] developed a hybrid algorithm to solve the measurement problem of 3D chemiluminescence intensity fields and validated this by both numerical and experimental methods. Despite the considerable progress, the reconstruction algorithm of CTC still has a great potential for further optimization in terms of computational speed, reconstruction precision, and efficiency by improving calculation models, access methods of the weight function, and the full use of the prior information. Gilabert et al. [11] and Hossain et al. [12] designed a series of experimental systems by using an optical transmission unit and optical fiber bundles, respectively, to increase the efficiency of CTC and reduce the cost. Fiberbased endoscopes (FBEs) were also applied for instantaneous 3D combustion measurements combined with tomography. Kang et al. [13] conducted a series of tests to quantify the accuracy of the view registration and the spatial resolution of the FBEs. These studies have further improved CTC by optimizing the reconstruction algorithm and designing the experimental system, which make CTC more suitable for combustion diagnostics. Numerical tests have been performed to evaluate the reconstruction algorithm. Most tests are performed on the assumption of parallel beams, which is uncertain in practical experiments as the depth of focus is limited, and thus more direct experimental verifications are needed to assess the feasibility and the accuracy of CTC. However, such convincing experiments are rarely reported.

Based on the development of the reconstruction algorithm and the experimental system, the CTC technique has been widely used in combustion diagnostics. Floyd et al. [14] reconstructed the $3 \mathrm{D}$ chemiluminescence field of a turbulent opposed jet flame with the CTC technique to obtain the flame surface density, wrinkling factor, flame normal direction, and heat release rate. They [15] also studied the instantaneous 3D information on flame geometry and excited species concentrations on a matrix burner using 10 simultaneous camera measurements. Anikin et al. [16] reconstructed 2D $\mathrm{OH}^{*}$ chemiluminescence distributions with a spatial resolution of about $1 \mathrm{~mm}$ in turbulent diffusion flames with an exposure time of $200 \mu \mathrm{s}$. Li [17] first obtained instantaneous 3D combustion structures of turbulent jet flames imaging across a sizable measurement volume at $\mathrm{kHz}$ based on tomographic chemiluminescence technique, and phantoms simulating the experimental flames were also performed for comparison and validation purposes [18]. Many reconstructed results of different flames have been obtained, but most studies just focus on the reconstruction of chemiluminescence field regardless of the relationship between the chemiluminescence information and the combustion characteristics.

The studies and applications of the CTC technique are still insufficient although considerable progress has been achieved in recent years. In this work, a phantom study is carried out to assess the ability of the ART algorithm. Then, a well-designed burner is employed to generate stable conical hydrogen-air premixed laminar flames, which are used to perform an experimental verification to further assess the feasibility of CTC. The $\mathrm{OH}^{*}$ chemiluminescence intensity fields, which are important indicators of reaction zones of flame fronts, are reconstructed using the CTC technique. With the use of these reconstructions, the flame structures and effects of flow velocities and equivalence ratios are studied.

\section{The CTC Method}

2.1. The ART Algorithm. The CTC method contains two main processes: acquisition of chemiluminescence projections from different angles and reconstruction of the intensity field of the chemiluminescence from these projections using a suitable reconstruction algorithm. The algebraic reconstruction technique (ART) algorithm is used in this study.

The ART algorithm is suitable for the CTC among a series of robust reconstruction algorithms due to these following reasons: the ART algorithm is a kind of iterative reconstruction algorithm, which is quite appropriate for the reconstruction from few projections and with severe noises; the ART algorithm allows for more flexible projection geometries, which brings great potential of applications for different flames; the ART algorithm is easy to be improved with a priori information, which greatly enhances the efficiency of the reconstruction. However, the ART algorithm also has some disadvantages, and the most serious one is its high dependence on computing resources. With the development of the computing devices, this problem is gradually solved, especially in 2D reconstructions. In this work, we only focus on $2 \mathrm{D}$ reconstructions and the most serious disadvantage of the ART algorithm is not a problem. Based on the reasons above, the ART algorithm is employed in this work.

The reconstruction problem is reduced to solving an underdetermined system of linear equations. The system is obtained by approximating each projection measurement $I_{q p}$ as a finite sum taken through the discrete object (the flame) domain as shown in the following [15]:

$$
I_{q p}=\sum_{v=1}^{N_{v}} w_{q p v} f_{v}
$$

where $I_{q p}$ is the intensity value of the $p$ th projection in the $q$ th view (an image contains a series of projections measured from a projection angle using a ICCD camera); $f_{v}$ is the average scalar value of the pixel $v$, which is the goal; $w_{q p v}$ is the weight coefficient representing the contribution of the pixel $v$ to the $p$ th (maximum value $N_{p}$ ) projection in the $q$ th (maximum value $N_{q}$ ) view, which is approximated as the proportion of the intersection area between the projection beam and the pixel area to the pixel area. With both the necessity to obtain the correct weight coefficient for each 
projection and the importance of enhancing the computing efficiency considered, a simplified calculation approach is employed in this study, where the pixel is divided into $10 \times$ 10 subgrids, and then the sum of area of these subpixels whose center passed through the beam is computed as the intersection area. In this work, the object domain is divided into pixels that are indexed by a single index $v$ from 1 to $N_{v}$.

A relaxed additive version of the ART algorithm is used in this work as follows [15]:

$$
F^{(i)}=F^{(i-1)}-\gamma \times \frac{\left(W_{i} \times F^{(i-1)}-I_{i}\right)}{W_{i} \times W_{i}^{T}} \times W_{i}^{T} .
$$

In this equation, $F$ is a $N_{v}$-dimensional vector of $f_{v} ; I$ is a $\left(N_{q} \times N_{p}\right)$-dimensional vector of $I_{q p} ; W$ is a $\left[\left(N_{q} \times N_{p}\right) \times N_{v}\right]$ dimensional matrix of $w_{q p v} ; \gamma$ is a relaxation factor, which is used to improve convergence in the presence of noises, and it is 0.5 in this work as the noise is not so high; index $i$ ( $i=$ $q \times p)$ represents both the index of the projection in all views and the number of the iteration. Once all indexes have been addressed, the next round of iteration begins.

A simple criterion described by Mishra et al. [19] is employed to reduce the computational cost. Once the absolute difference of the sum of the chemiluminescence intensity $\left(f_{v}\right)$ is below a threshold value $0.0001 \times\left|F^{i}\right|$, the reconstruction is considered to be converged.

In this work, the $2 \mathrm{D}$ reconstruction domain was divided into 131072 pixels $\left(N_{v}=131072\right) .37$ projection angles $\left(N_{q}=\right.$ 37 ) were chosen, and the number of projections in each view was $255\left(N_{p}=225\right)$ as the amount of the reconstruction grids was small.

2.2. Phantom Study. Phantom studies are typically used to assess the feasibility and the accuracy of the reconstruction algorithm, which is the foundation of the CTC technique. A phantom object is designed with a size of $1 \times 0.5$ (relative length with unit of 1 , as the length unit is meaningless in a phantom study) as shown in Figure 1, which is divided into $200 \times 100$ pixels and the detailed parameters are listed in Table 1. It includes four geometric shapes of one rectangle and three intersecting rings. This phantom object is similar to the shape of the flame structure, used in our experimental studies. The corners of the rectangle can be used to assess the abilities of the ART algorithm to reconstruct detailed information and the intersecting rings can help to assess the spatial resolution.

Projections were achieved in every 5 degrees from 0 degrees (the angle of the projection orientation to the base of the phantom object) to 180 degrees. Up to 37 views in total were collected, and each view included 225 projections. The criterion was followed strictly.

Through the analysis of the flow field and the experience of previous work, the relative position of the flame can be determined before the reconstruction. This will be greatly helpful to improve the accuracy of the reconstruction. The method is known as the introduction of prior knowledge. It was implemented in this work. Figure 2 shows an initial distribution of the reconstruction, which is determined by the prior knowledge. The detailed parameters are listed in Table 2.

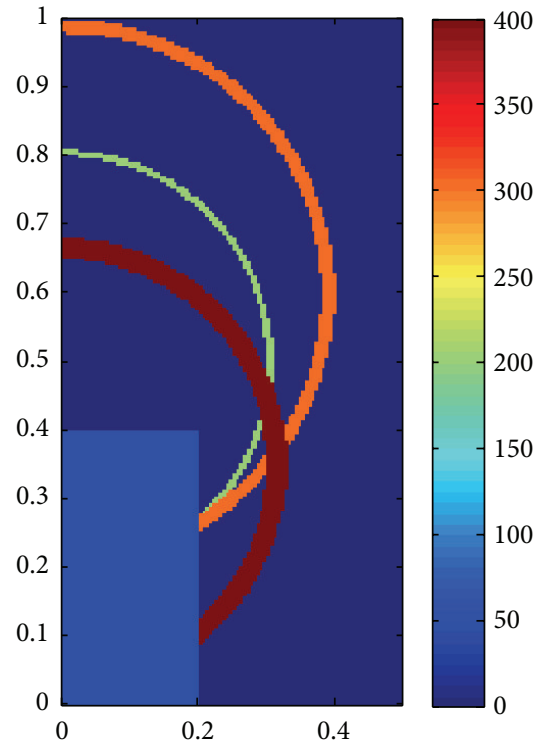

FIGURE 1: The phantom object for testing the reconstruction algorithm.

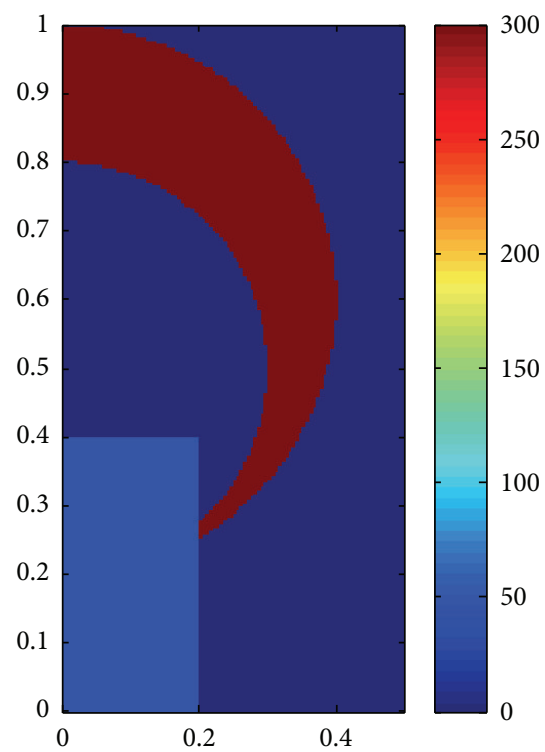

FIGURE 2: The initial distribution of the reconstruction.

TABLE 1: Geometric parameters of the phantom object.

\begin{tabular}{lcccc}
\hline Shape & $\begin{array}{c}\text { Width } \\
\text { (in-radius) }\end{array}$ & $\begin{array}{c}\text { Length } \\
\text { (out-radius) }\end{array}$ & $\begin{array}{c}\text { Center } \\
\text { coordinate }\end{array}$ & Intensity \\
\hline Rectangular & 0.20 & 0.40 & $(0.10,0.20)$ & 50 \\
Ring & 0.30 & 0.31 & $(0.00,0.50)$ & 200 \\
Ring & 0.38 & 0.40 & $(0.00,0.60)$ & 300 \\
Ring & 0.30 & 0.33 & $(0.00,0.35)$ & 400 \\
\hline
\end{tabular}

The reconstructed results are shown in Figure 3, which shows a good agreement with the phantom object in general distribution information of the geometric shapes, but small 


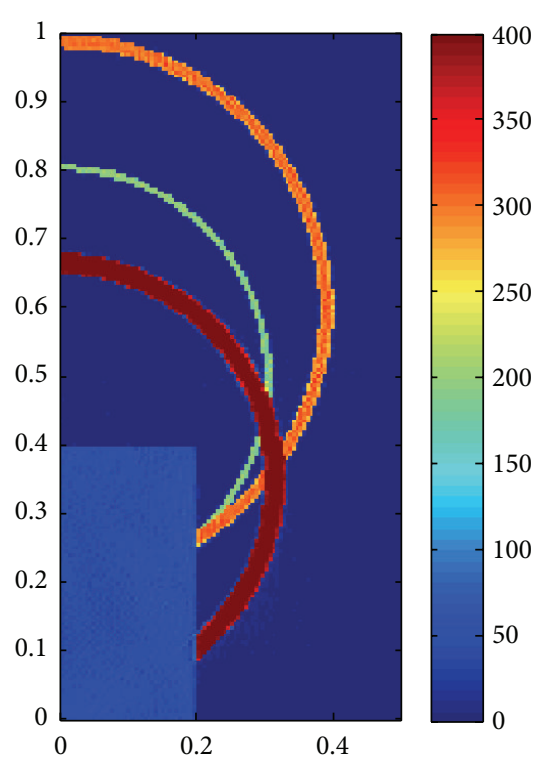

FIGURE 3: The reconstructed result of the phantom object.

TABLE 2: Geometric parameters of the initial distribution.

\begin{tabular}{lcccc}
\hline Shape & $\begin{array}{c}\text { Width } \\
\text { (in-radius) }\end{array}$ & $\begin{array}{c}\text { Length } \\
\text { (out-radius) }\end{array}$ & $\begin{array}{c}\text { Center } \\
\text { coordinate }\end{array}$ & Intensity \\
\hline Rectangular & 0.20 & 0.40 & $(0.10,0.20)$ & 50 \\
Ring & 0.30 & & $(0.00,0.50)$ & 300 \\
Ring & & 0.40 & $(0.00,0.60)$ & 300 \\
\hline
\end{tabular}

noises can be seen and the detailed information has some errors.

In order to further assess the accuracy of the ART algorithm, the maximum deviation coefficient $E_{m}$ and the normalized root mean square error (NRMSE) $E_{n}$ were calculated. They are defined as follows, respectively:

$$
\begin{gathered}
E_{m}=\frac{\max (F)}{f_{v \max }}, \\
E_{n}=\sqrt{\frac{\sum_{v=1}^{N_{v}}\left(F_{v}-f_{v}\right)^{2}}{\sum_{v=1}^{N_{v}}\left(f_{v}\right)^{2}}},
\end{gathered}
$$

where $F$ is a $N_{v}$-dimensional vector of the reconstructed result; $f_{v}$ is the average scalar value of the pixel $v ; f_{v \max }$ is the average scalar value in the same pixel with the maximum element of $F . E_{m}$ represents the maximum deviation between the phantom object and the reconstruction. It indicates the worst situation in the reconstruction. $E_{n}$ represents a general deviation level. These two coefficients can effectively assess the accuracy of the reconstructions and verify the feasibility of the ART algorithm.

Relative intensity distributions (at different heights above the bottom) were compared as shown in Figure 4 at three different relative heights of $0.25,0.50$, and 0.75 from the bottom to the top, respectively. The relative intensities, which

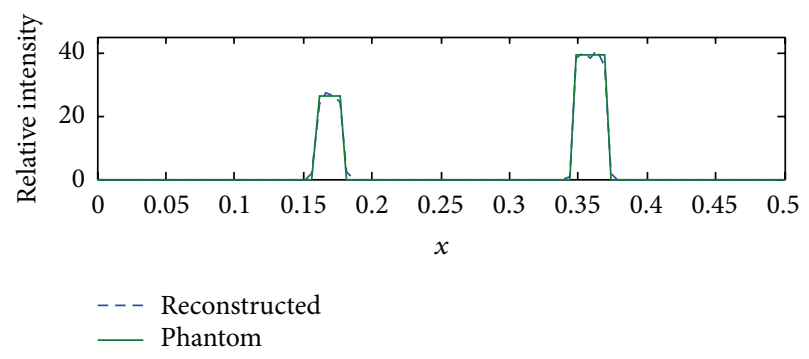

(a)

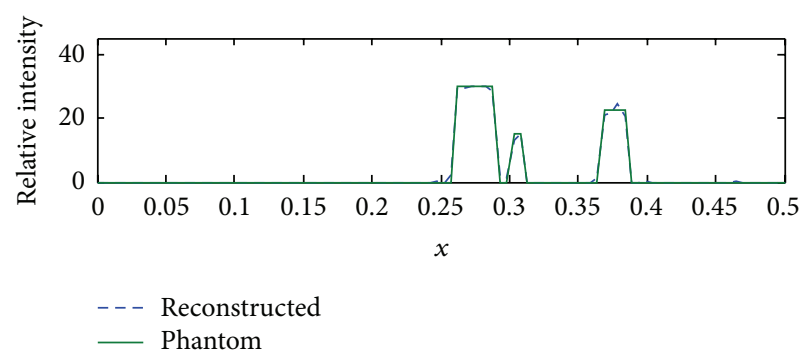

(b)

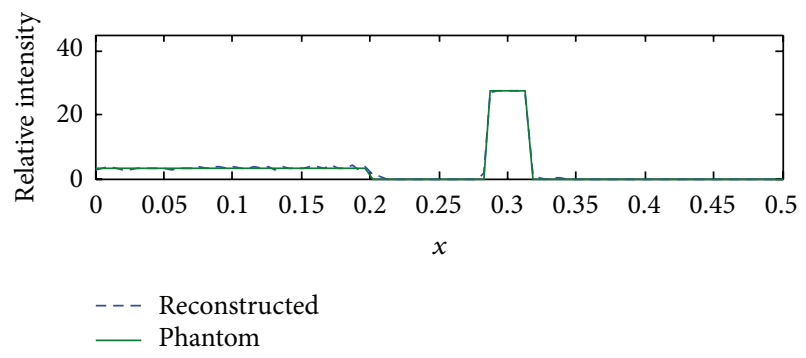

(c)

FIGURE 4: Comparison of relative intensity distributions of the phantom object and the reconstructed result at three different relative heights of $0.25,0.50$, and 0.75 from the bottom to the top (the axis " $x$ " represents the relative distance to the left boundary of the phantom).

were achieved from the intensity values divided by the average values on each height, showed a good agreement with each other. In this phantom, $E_{n}$ is calculated as 0.059 , which is a very small one. It quantitatively represents a good performance of the ART algorithm. However, in the areas of large intensity gradient, errors can be found up to $9.3 \%\left(E_{m}\right.$ is 0.093), which indicates a weakness of the ART algorithm in reconstructions of detailed information. In spite of this, the reconstructed result indicated main features of the structure of the phantom object, which verify the feasibility of the ART algorithm.

\section{Experimental Arrangement}

3.1. Combustion System. The burner and its gas supply system used in this study are illustrated in Figure 5(a). The burner had two blocks with the height of $15 \mathrm{~mm}$, which were arranged on the perforated plate with a distance of $2 \mathrm{~mm}$. It mainly consisted of the premix chamber, flame arrester device, perforated plate, and protective gas chamber. The 

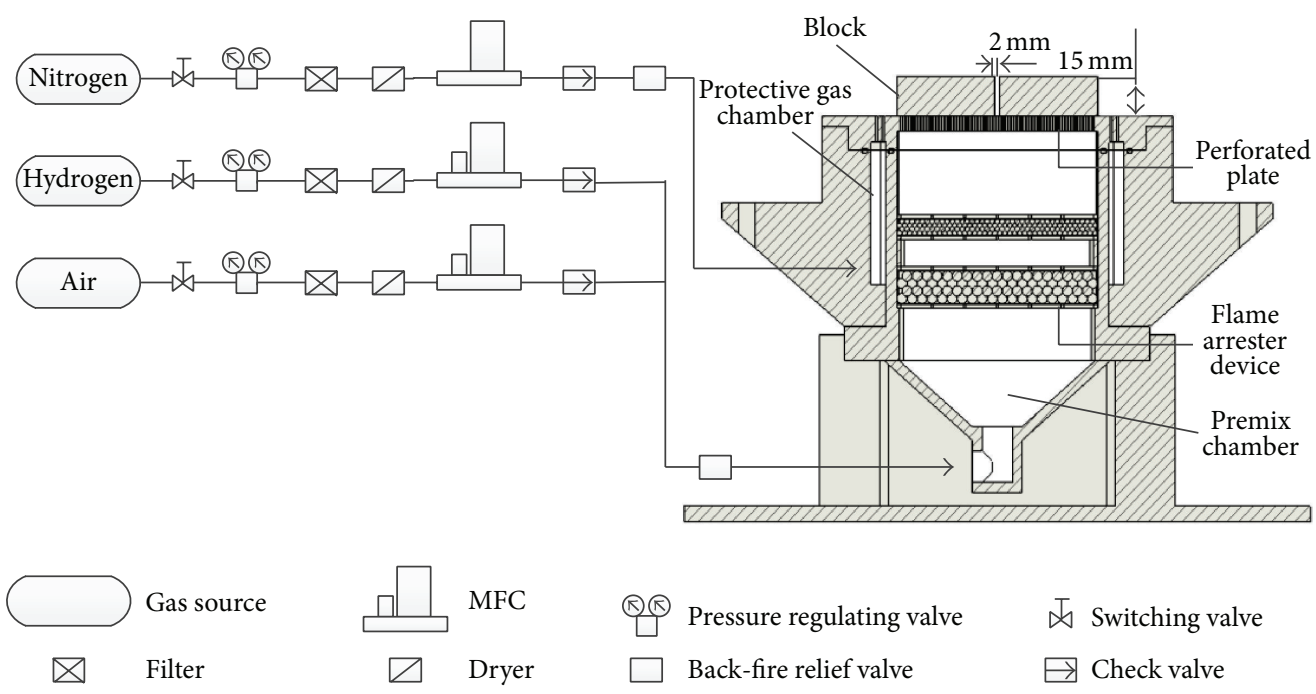

(a)
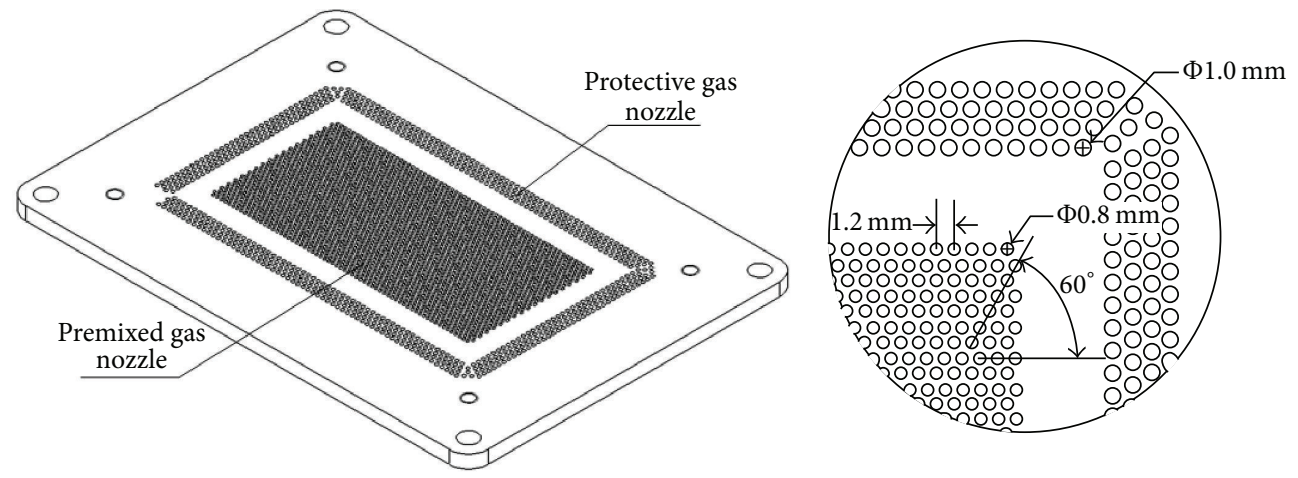

(b)

Figure 5: Schematics of the combustion system: (a) burner and its gas supply system; (b) arrangement of the circular nozzles.

arrangement of the circular nozzles on perforated plate was shown in Figure 5(b). Flame arrester device mounted in the premix chamber mainly consisted of steel balls with two different diameters of $2 \mathrm{~mm}$ and $1 \mathrm{~mm}$, respectively, to get rid of backfire. Check valves and backfire relief valves were also employed in the pipelines for safety reason. Gases were adjusted by Alicat Scientific mass flow controllers (MFCs), which could provide adequate flow control with high accuracy. Premixed reactants (hydrogen-air) entered the burner and were further mixed in the premix chamber to ensure the accuracy of the equivalence ratio of the reactant stream above the blocks at the burner exit. $\mathrm{N}_{2}$ was used as a protective gas to provide a relatively closed region eliminating the interference of the ambient air. Stable hydrogen-air premixed laminar flames, which were perfect for the verification experiment, were achieved due to the special structure of the burner.

In this work, 9 different flame conditions were designed to study the effects of the flow velocity and the equivalence ratio on the flame structure. Considering the stability of the flames, the flow velocities were limited from $11 \mathrm{~m} / \mathrm{s}$ to $15 \mathrm{~m} / \mathrm{s}$ and the equivalence ratios were changed from 0.35 to 0.55 , as shown in Table 3.
TABLE 3: A list of 9 flame conditions with different flow velocities and equivalence ratios.

\begin{tabular}{lcc}
\hline Identifier & Equivalence ratio & Flow velocity $(\mathrm{m} / \mathrm{s})$ \\
\hline F1 & 0.45 & 14.36 \\
F2 & 0.45 & 13.04 \\
F3 & 0.45 & 11.73 \\
F4 & 0.45 & 15.67 \\
F5 & 0.45 & 16.95 \\
F6 & 0.50 & 14.36 \\
F7 & 0.55 & 14.36 \\
F8 & 0.40 & 14.36 \\
F9 & 0.35 & 14.36 \\
\hline
\end{tabular}

3.2. Optical System. Figure 6(a) illustrates two orientations of the projection view and the side view. The projection view orientation demonstrated a plane, in which projections were obtained, while the side view orientation demonstrated an orientation perpendicular to the projection plane in which integral images were recorded directly. Integral images of the $\mathrm{OH}^{*}$ chemiluminescence from the side view were used as a 


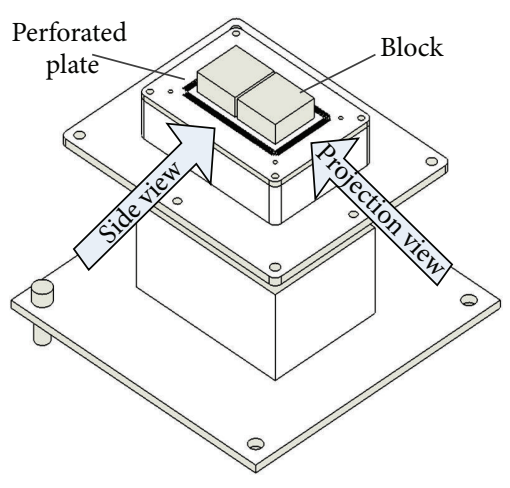

(a)

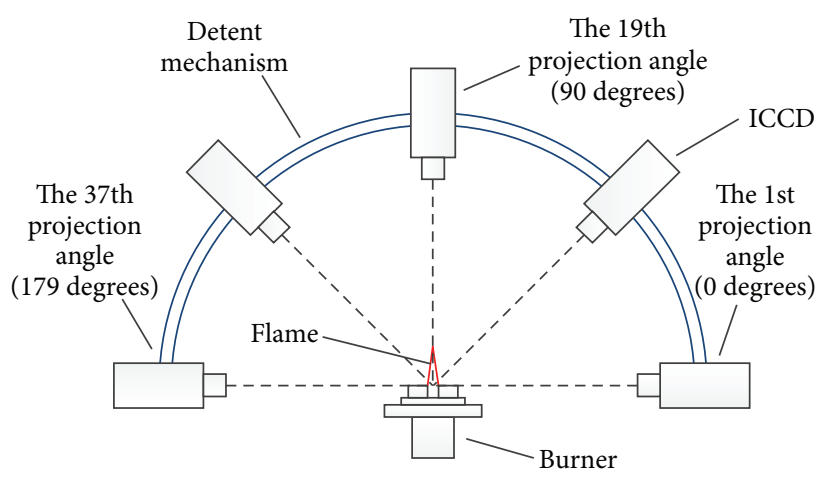

(b)

Figure 6: Schematics of the optical system: (a) two typical view orientations; (b) a single camera mounted on a guideway and rotated around a fixed center.

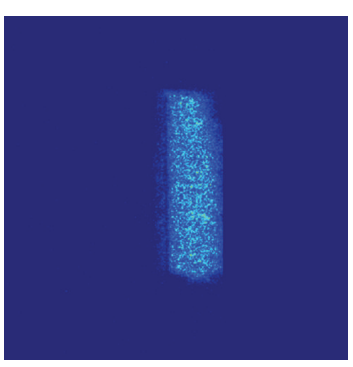

$0^{\circ}$

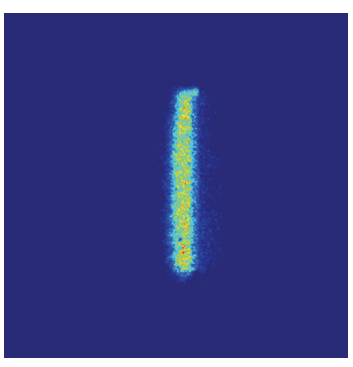

$90^{\circ}$

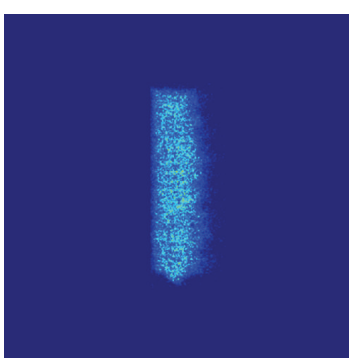

$45^{\circ}$

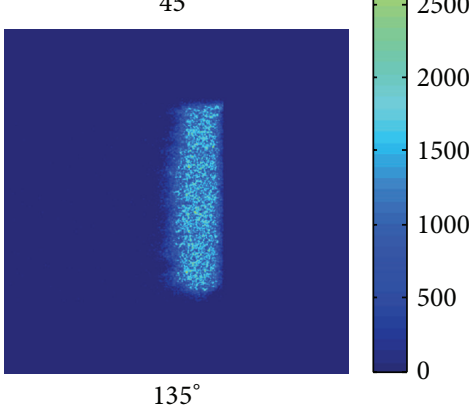

FIGURE 7: Projection images from four projection angles of $0,45,90$, and 135 degrees.

real 2D distribution of the $\mathrm{OH}^{*}$ chemiluminescence for comparison with the reconstructed results. An annular guideway working as a detent mechanism was mounted vertically to the burner exit located at the center, as shown in Figure 6(b). A single camera was mounted on the guideway and rotated around the center, while maintaining the alignment and the distance of $750 \mathrm{~mm}$ from the center. Every 5 degrees from 0 degrees to 180 degrees, added up to a total of 37 angles, were chosen as projection angles. Due to symmetric view, the projections from 0 degrees and 180 degrees were identical, of which the angle of 179 degrees was chosen to be the 37 th projection angle as a result. Views were measured from 37 projection angles as mentioned above using a PI-MAX I $512 \times$ 512-pixel ICCD camera with a $50 \mathrm{~ms}$ exposure time and a gain of 20, which were appropriate for getting a high signal-tonoise ratio (SNR). An interference band-pass filter of $307 \mathrm{~nm}$ with $17 \%$ transmission at peak frequency and a full width at half maximum of $12 \mathrm{~nm}$ was added in front of the UV lens for acquiring $\mathrm{OH}^{*}$ chemiluminescence.

Calibration was carried out to ensure the precise location of the center, along which the camera rotated. The boundaries of the chemiluminescence images were easily recognized as the shape of the flame in this study was simple. The midline of the boundaries was taken as the reconstruction plane (which here was a line in an image) in each view, which was also helpful to get the projection geometries.

Figure 7 shows 4 images (views) from the projection angles of $0,45,90$, and 135 degrees. The midline of the boundaries of the chemiluminescence distribution in each of the images was used as the projections for reconstruction as mentioned above.

\section{Results and Discussion}

4.1. Experimental Verification for CTC. The reconstructed result obtained using the CTC technique is presented in Figure 8(a), along with the side image (the integral image achieved from the side view as mentioned above) shown in Figure $8(\mathrm{~b})$. The side image was processed by position transformation to make it easier to compare. It is clear that the reconstructed result can reflect the distributions of the $\mathrm{OH}^{*}$ chemiluminescence of the flame despite the slight differences in detail.

In order to further evaluate the feasibility of CTC, a relative intensity distribution is compared between two images in Figure 8. Figure 9 shows the comparison at three different heights above the exit plane of $2 \mathrm{~mm}, 6 \mathrm{~mm}$, and $10 \mathrm{~mm}$, respectively. As shown in Figure 8, the relative intensities agree well with each other, and $E_{n}$ is calculated as 0.185 . Differences can be found especially on the bottom part with the maximum deviation up to $26.7 \%$, which shows a weakness of the ART algorithm to reconstruct the field with a cavity surrounded by the valuable information (areas of large intensity gradient). In spite of this, the reconstructed result can indicate main features of the flame structures, which verify the feasibility of CTC. 


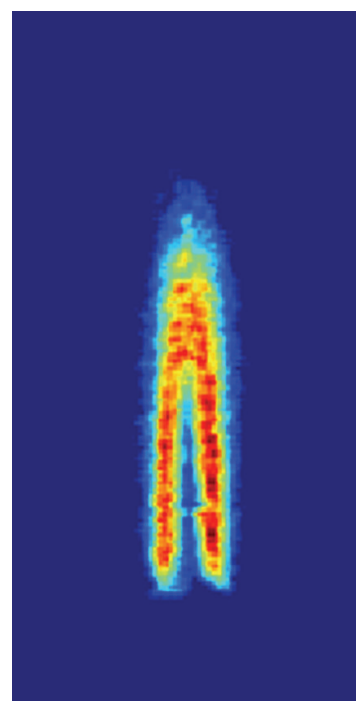

(a)

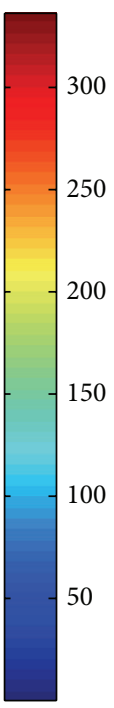

200

150

100

50

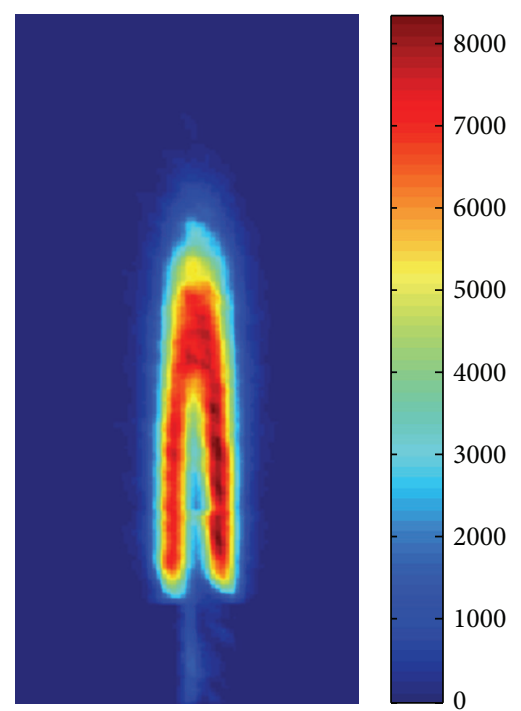

(b)

FIGURE 8: Comparison of the reconstructed result (a) and the side image (b) of the $\mathrm{OH}^{*}$ chemiluminescence.

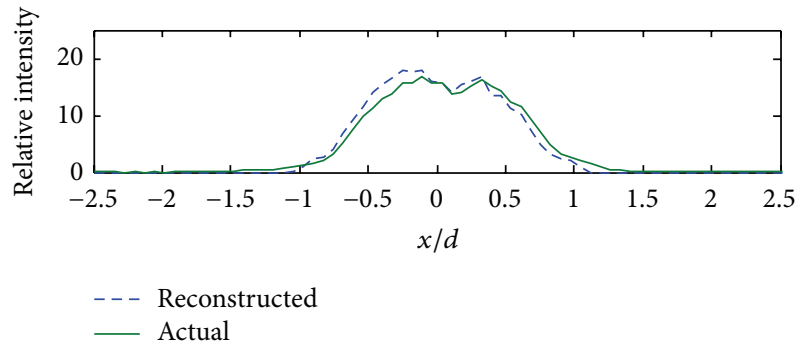

(a)

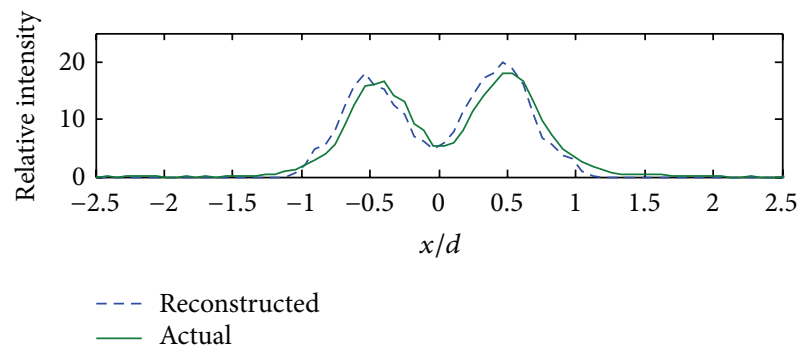

(b)

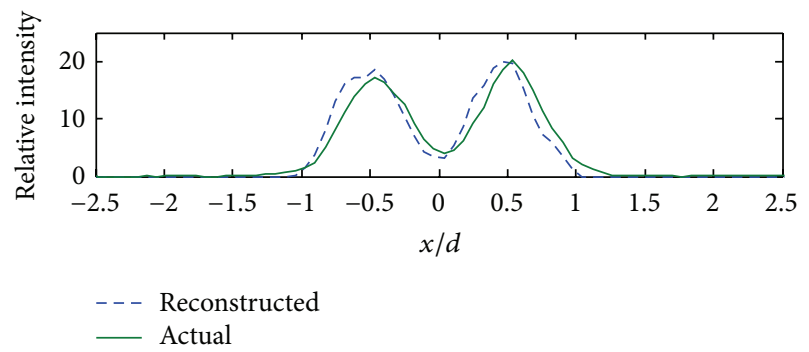

(c)

FIGURE 9: Comparison of relative intensity distributions of the reconstructed results and the side image at three different heights of 2,6 , and $10 \mathrm{~mm}$ from the bottom to the top (the axis " $x / d$ " represents the ratio of the distance to the center of the burner and the width of the exit).
The coefficients $\left(E_{n}\right.$ and $\left.E_{m}\right)$ in this reconstruction are bigger than those in the phantom study by about 3.1 times and 2.9 times, respectively. This is mainly caused by the experiment devices and the errors in projecting system. The uncertainty of this CTC system for combustion diagnostics is determined by the maximum deviation (26.7\% in this work). It is a big one for quantitative analysis. However, the relative distribution of the $\mathrm{OH}^{*}$ chemiluminescence in flames are fairly precise. It is good enough to perform the study of the flame structures by the CTC technique.

4.2. Flame Structures Based on CTC. Figure 10 shows the reconstructions of the $\mathrm{OH}^{*}$ chemiluminescence for 8 flame conditions. A blow-off phenomenon appeared in F9 and the reconstructed result was not displayed as no $\mathrm{OH}^{*}$ chemiluminescence was obtained in this condition. These reconstructed results of the $\mathrm{OH}^{*}$ chemiluminescence intensity fields can represent the real $\mathrm{OH}^{*}$ radiation distributions. $\mathrm{OH}^{*}$ distribution is an important indicator of the reaction zones of the flame fronts and it can characterize the flame structure. The intensity values are increasing with larger flow velocities as shown in Figure 10, due to the increasing reactant mixtures. Figure 10 also shows the same trend with the increasing equivalence ratios.

In order to characterize the flame structure information, the height of the flame (HF) and the ratio of relative distributions of the flame chemiluminescence (RDF) were employed in the $\mathrm{OH}^{*}$ data analysis. The height of the flame (HF) is defined as

$$
\mathrm{HF}=Y_{d}-Y_{e}
$$

where $Y_{d}$ is the vertical coordinate of the dividing line between the flame and the surroundings determined by the threshold value, which is $1 / 3$ peak value in each image, and $Y_{e}$ is the vertical coordinate of the burner exit. The ratio of 


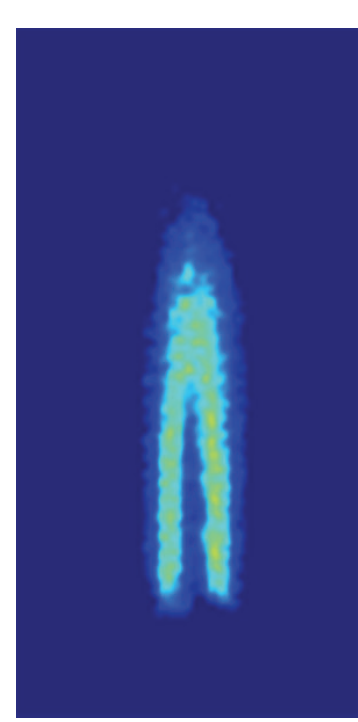

F1

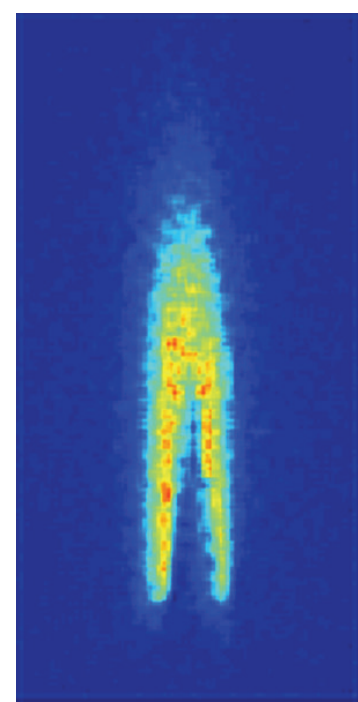

F5

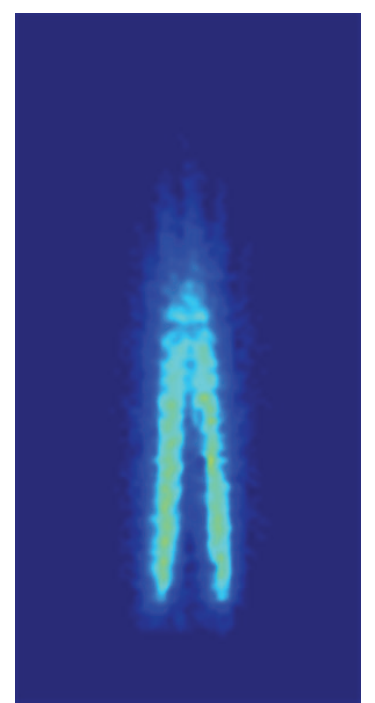

F2

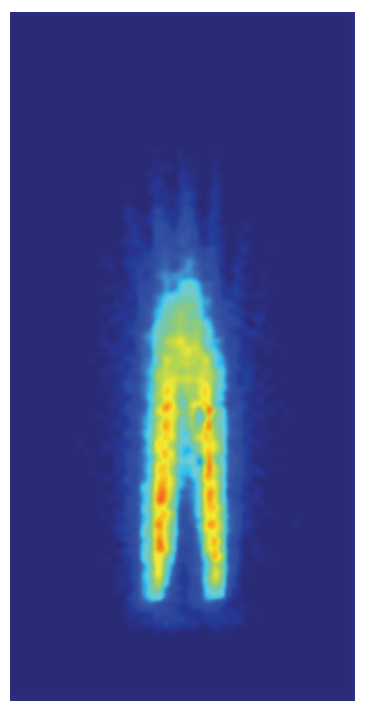

F6

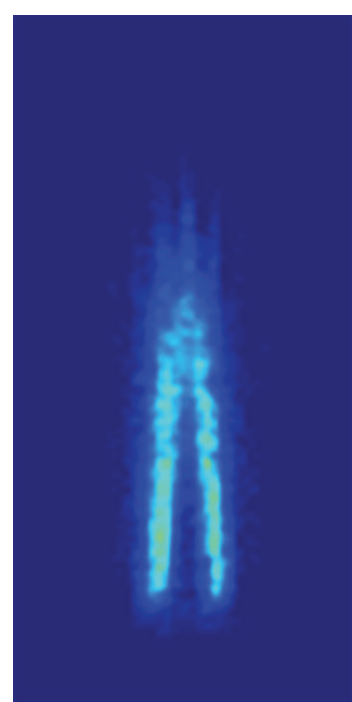

F3

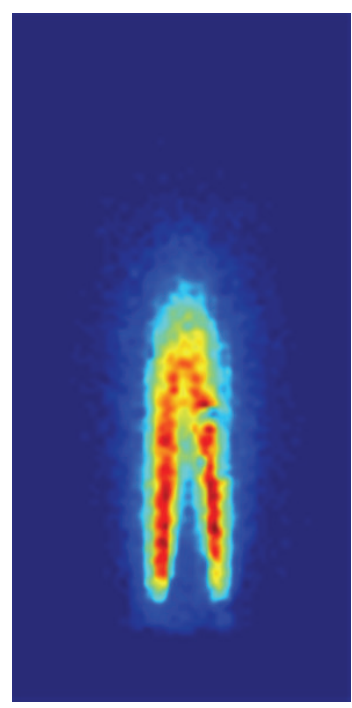

F7

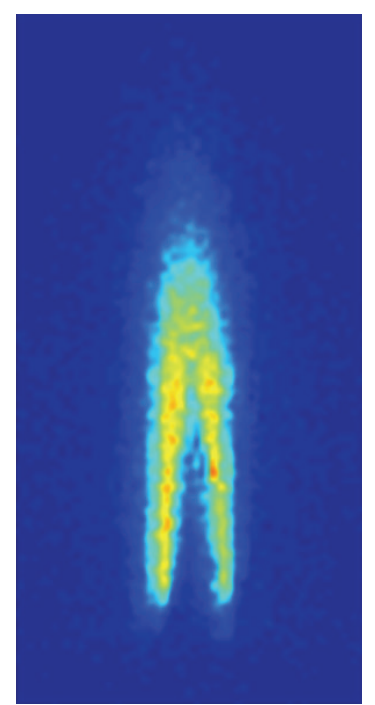

F4

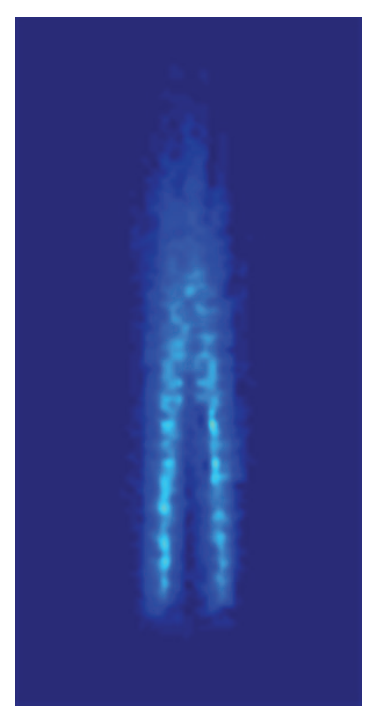

F8

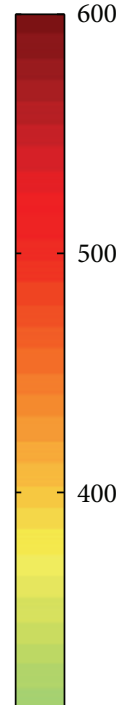

300

200

100

Figure 10: Reconstructions of the $\mathrm{OH}^{*}$ chemiluminescence in different flame conditions.

relative distributions of the flame chemiluminescence (RDF) is defined as

$$
\mathrm{RDF}=\frac{I_{\mathrm{ts}}}{I_{\mathrm{ws}}},
$$

where $I_{\mathrm{ts}}$ is the sum of the chemiluminescence intensities of the top half of the flame; $I_{\mathrm{ws}}$ is the sum of the chemiluminescence intensities of the whole of the flame. Flame structures can be preliminarily characterized by these two parameters.

HFs and RDFs with different flow velocities and equivalence ratios are presented in Figure 11. HF becomes larger with increasing flow velocities as shown in Figure 11(a), whereas it decreases with increasing equivalence ratios (no larger than 1) as shown in Figure 11(b). HF relies on the relative relations between the flow velocity and the flame propagation velocity, which is connected with the equivalence ratio. The largest flame propagation velocity is usually obtained near the equivalence ratio of 1 with other parameters unchanged. That means a larger flame propagation velocity with a larger equivalence ratio (no larger than 1) in this study. Figure 12 shows a geometric relationship of the flame structure and the velocities. It is clear that HF is proportional to $\tan \theta$, which is determined by the flow velocity and the flame propagation velocity. It shows the same regularities with the experimental results. The 9th flame condition shows a blowoff phenomenon, as the cone angle is too sharp to hold the flame. Therefore, the cone angle of 4.76 degrees, which is calculated by HF and the exit width, in 8th flame condition can be considered a critical cone angle, which will be a useful criterion to avoid the blow-off phenomenon, although the actual one should be sharper.

Figure 11(a) shows a positive effect of flow velocities on RDFs, indicating that the reaction zone was gradually lifted with increasing flow velocities. The equivalence ratio shows 


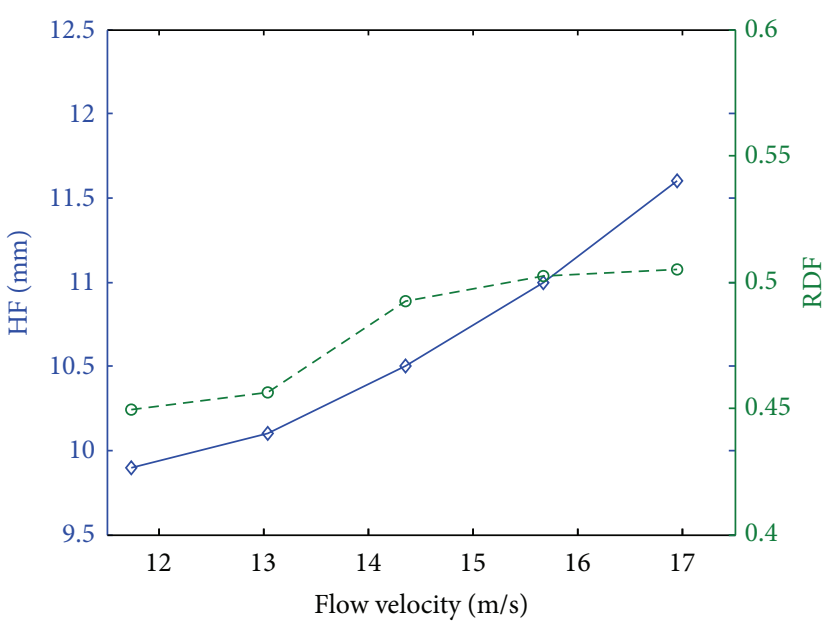

$\rightarrow$ HF

$-\theta-\mathrm{RDF}$

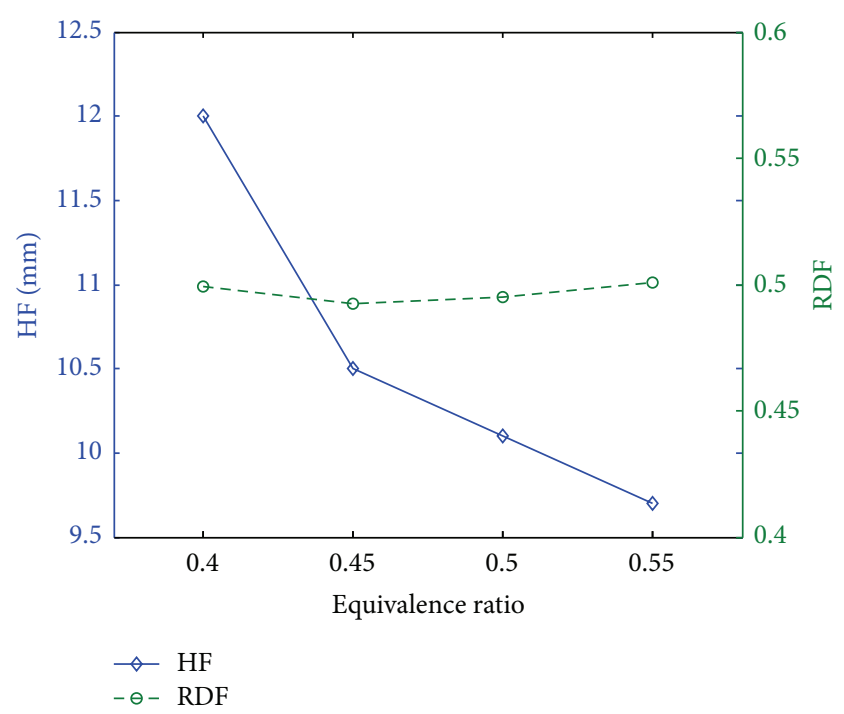

(b)

FIGURE 11: HFs and RDFs in different flame conditions: (a) flow velocities; (b) equivalence ratios.

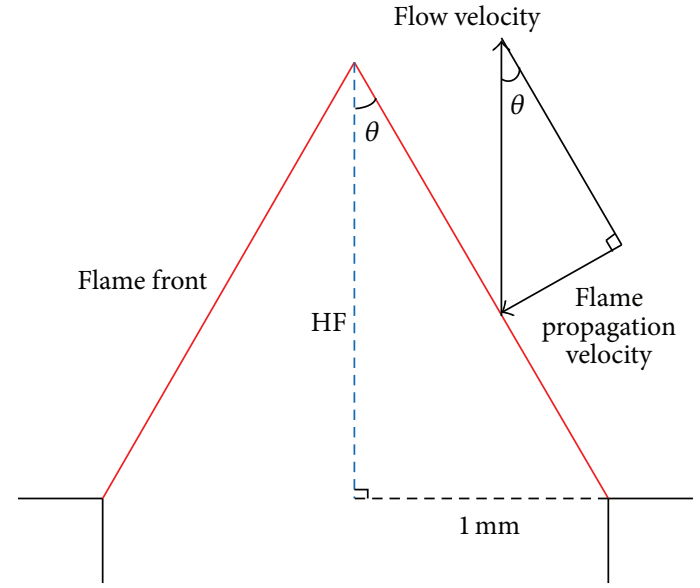

FIgURE 12: Geometric relationships of the flame structure and the velocities.

a minor effect as seen in Figure 11(b). Therefore, RDF is independent of HF and is just related to the flow velocity, suggesting RDF is a useful parameter of the flame structure. Figure 11(a) also reveals a critical flow velocity of about $15 \mathrm{~m} / \mathrm{s}$, which leads RDF to 0.5 . It is an indicator of the $\mathrm{OH}^{*}$ chemiluminescence distributions. It suggests that the reactions relatively gather to the top of the flame and the flame gradually separates from the exit of the burner, while the flow velocity increases to $15 \mathrm{~m} / \mathrm{s}$. It should be noted that this is just a rough estimate. Some other factors may also contribute to RDF, which calls for more experimental data and further studies.

\section{Conclusions}

In conclusions, the numerical and experimental verifications of computed tomography of chemiluminescence (CTC) were conducted and the structures of hydrogen-air premixed laminar flames were investigated using CTC. A phantom study was performed to assess the ability of the ART algorithm. A well-designed burner was built to generate a stable hydrogen-air premixed laminar flame for the experimental verification of CTC. The experimental verification was carried out by comparing the reconstructed result to the side image recorded directly from the side view. The $\mathrm{OH}^{*}$ chemiluminescence intensity fields reconstructed by CTC were used to indicate the flame structures and study the effects of flow velocities and equivalence ratios on the flame structure. Detailed conclusions obtained from this study are listed as follows.

The uncertainty of this CTC system for combustion diagnostics is determined as $26.7 \%$ in this work, and it is not suitable for quantitative analysis. However, the reconstructed results can still effectively indicate the relative distributions of the flame chemiluminescence. It is good enough to perform the study of the flame structures by the CTC technique.

The height of the flame is affected by the flow velocity and the equivalence ratio. With an increase of HF, the cone angle of the flame structure decreases until a blow-off phenomenon appears at a critical cone angle of 4.76 degrees.

The increasing flow velocities gradually lift the reaction zone, while the equivalence ratio has a minor effect on it.

It should be noted that the CTC technique still fails to show the flame structure with high accuracy in areas with a large intensity gradient, which calls for further optimizations. In addition, the extension of the CTC method to reconstruct complex 3D turbulent flames is also required in the next step.

\section{Competing Interests}

There are no competing interests related to this paper. 


\section{Acknowledgments}

The authors would like to express their thanks for the support from the National Natural Science Foundation of China (Grants nos. 11272351 and 91441121).

\section{References}

[1] A. G. Gaydon, H. G. Wolfhard, and R. M. Fristrom, Flames: Their Structure, Radiation and Temperature, Chapman and Hall, London, UK, 1978.

[2] K. Kuwahara and H. Ando, "Analysis of barrel-stratified leanburn flame structure by two-dimensional chemiluminescence measurement," JSME International Journal B, vol. 37, no. 3, pp. 650-658, 1994.

[3] J. Kojima, Y. Ikeda, T. Nakajima et al., "Chemiluminescencebased diagnostics for the flame-front structure of premixed flames," Transactions of the Japan Society of Mechanical Engineers Part B, vol. 69, no. 678, pp. 482-489, 2003.

[4] M. Röder, T. Dreier, and C. Schulz, "Simultaneous measurement of localized heat-release with $\mathrm{OH} / \mathrm{CH}_{2} \mathrm{O}$-LIF imaging and spatially integrated $\mathrm{OH}^{*}$-chemiluminescence in turbulent swirl flames," Proceedings of the Combustion Institute, vol. 34, no. 2, pp. 3549-3556, 2013.

[5] Y. K. Jeong, C. H. Jeon, and Y. J. Chang, "Evaluation of the equivalence ratio of the reacting mixture using intensity ratio of chemiluminescence in laminar partially premixed $\mathrm{CH} 4$-air flames," Experimental Thermal and Fluid Science, vol. 30, no. 7, pp. 663-673, 2006.

[6] B. Taupin, G. Cabot, G. Martins, D. Vauchelles, and A. Boukhalfa, "Experimental study of stability, structure and $\mathrm{CH}^{*}$ chemiluminescence in a pressurized lean premixed methane turbulent flame," Combustion Science and Technology, vol. 179, no. 1-2, pp. 117-136, 2007.

[7] H. M. Hertz and G. W. Faris, "Emission tomography of flame radicals," Optics Letters, vol. 13, no. 5, pp. 351-362, 1988.

[8] N. Denisova, P. Tretyakov, and A. Tupikin, "Emission tomography in flame diagnostics," Combustion and Flame, vol. 160, no. 3, pp. 577-588, 2013.

[9] A. Goyal, S. Chaudhry, and P. M. V. Subbarao, "Direct three dimensional tomography of flames using maximization of entropy technique," Combustion and Flame, vol. 161, no. 1, pp. 173-183, 2014.

[10] W. Cai, X. Li, F. Li, and L. Ma, "Numerical and experimental validation of a three-dimensional combustion diagnostic based on tomographic chemiluminescence," Optics Express, vol. 21, no. 6, pp. 7050-7064, 2013.

[11] G. Gilabert, G. Lu, and Y. Yan, "Three-dimensional tomographic reconstruction of the luminosity distribution of a combustion flame," IEEE Transactions on Instrumentation and Measurement, vol. 56, no. 4, pp. 1300-1306, 2007.

[12] M. M. Hossain, G. Lu, and Y. Yan, "Three-dimensional reconstruction of combustion flames through optical fiber sensing and CCD imaging," in Proceedings of the 2011 IEEE International Instrumentation and Measurement Technology Conference (I2MTC '11), pp. 79-83, Binjiang, China, May 2011.

[13] M. W. Kang, X. Li, and L. Ma, "Three-dimensional flame measurements using fiber-based endoscopes," Proceedings of the Combustion Institute, vol. 35, no. 3, pp. 3821-3828, 2015.

[14] J. Floyd, P. Geipel, and A. M. Kempf, "Computed Tomography of Chemiluminescence (CTC): instantaneous 3D measurements and Phantom studies of a turbulent opposed jet flame," Combustion and Flame, vol. 158, no. 2, pp. 376-391, 2011.

[15] J. Floyd and A. M. Kempf, "Computed Tomography of Chemiluminescence (CTC): high resolution and instantaneous 3-D measurements of a matrix burner," Proceedings of the Combustion Institute, vol. 33, no. 1, pp. 751-758, 2011.

[16] N. B. Anikin, R. Suntz, and H. Bockhorn, "Tomographic reconstruction of $2 \mathrm{D}-\mathrm{OH}^{*}$-chemiluminescence distributions in turbulent diffusion flames," Applied Physics B, vol. 107, no. 3, pp. 591-602, 2012.

[17] X. Li, “Three-dimensional measurements of turbulent jet flames at $\mathrm{KHz}$ rate based on tomographic chemiluminescence," in Proceedings of the Aerospace Sciences Meeting, Reno, Nev, USA, January 2000.

[18] X. Li and L. Ma, "Volumetric imaging of turbulent reactive flows at $\mathrm{kHz}$ based on computed tomography," Optics Express, vol. 22, no. 4, pp. 4768-4778, 2014.

[19] D. Mishra, J. P. Longtin, R. P. Singh, and V. Prasad, "Performance evaluation of iterative tomography algorithms for incomplete projection data," Applied Optics, vol. 43, no. 7, pp. 1522-1532, 2004. 


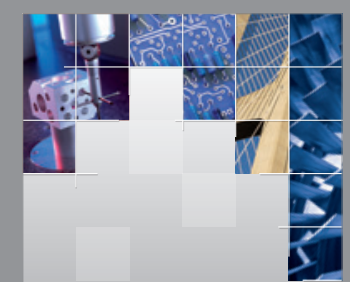

\section{Enfincering}
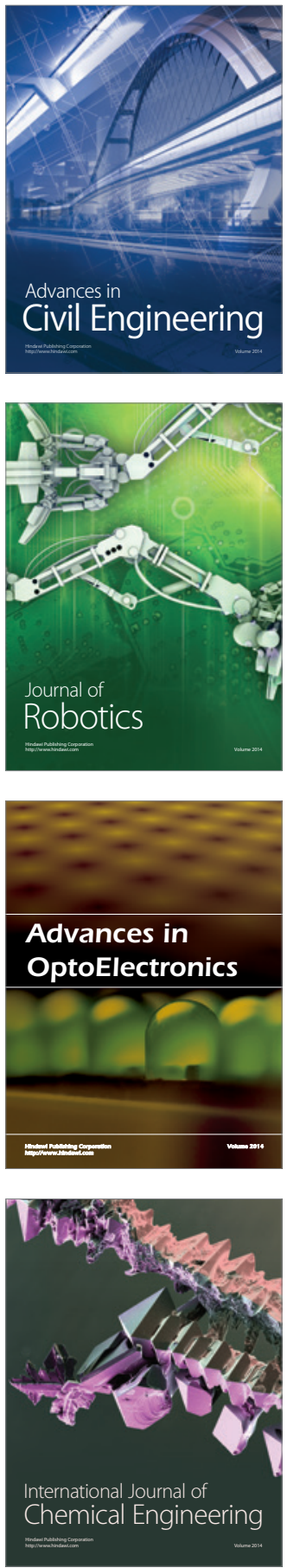

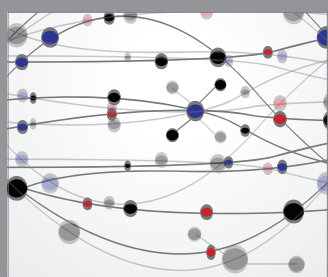

The Scientific World Journal

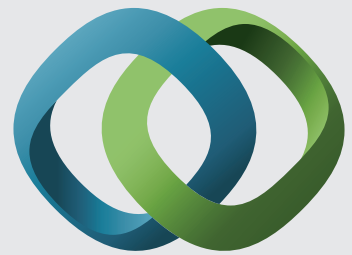

\section{Hindawi}

Submit your manuscripts at

http://www.hindawi.com
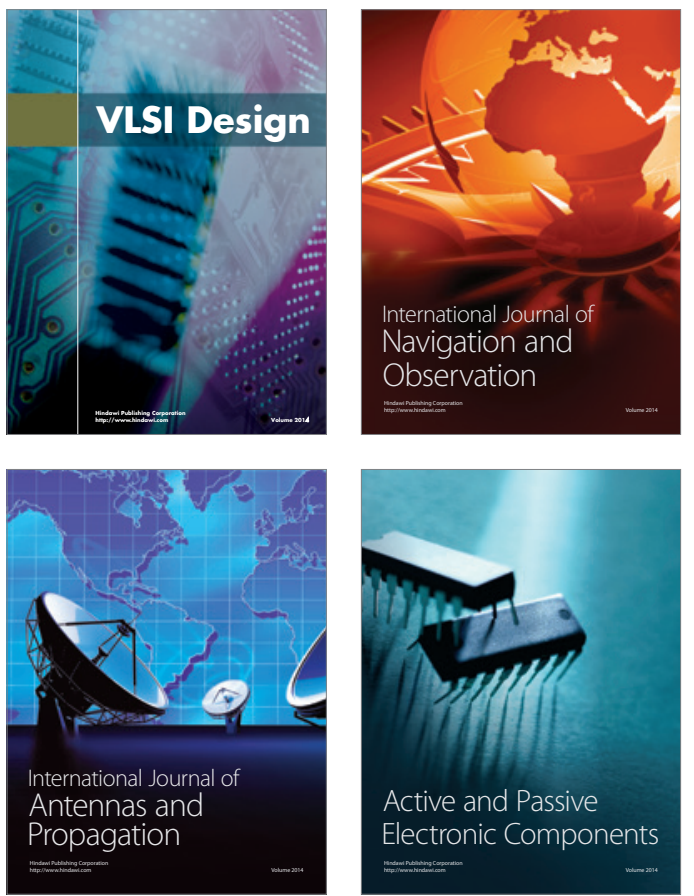
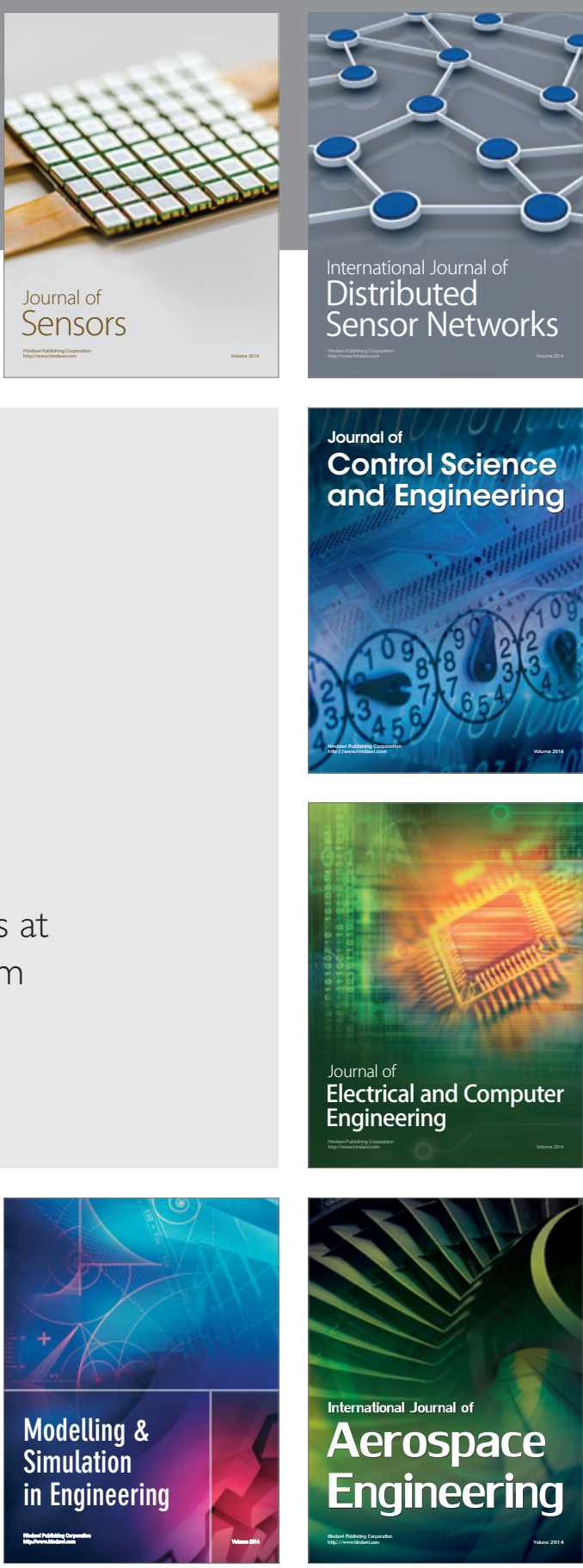

International Journal of

Distributed

Sensor Networks

Journal of

Control Science

and Engineering
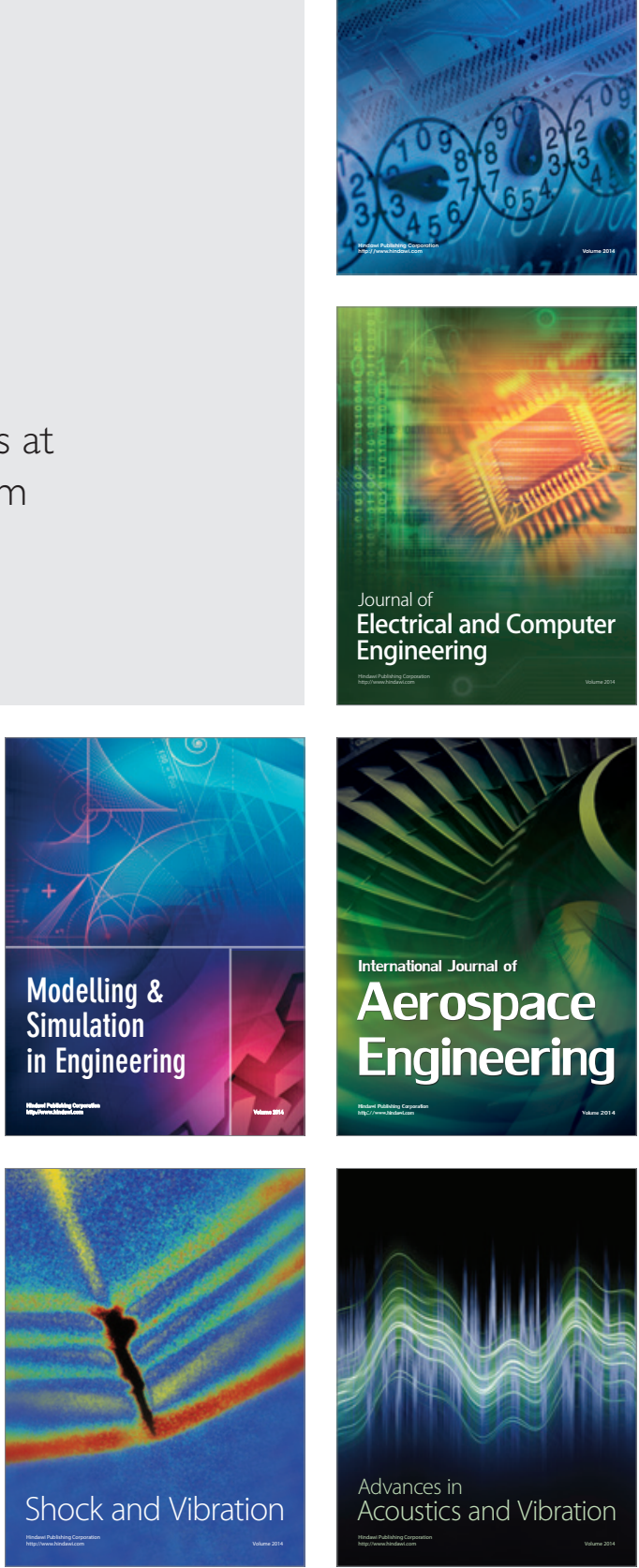